\title{
ON VARIOUS DEFINITIONS OF CAPACITY AND RELATED NOTIONS
}

\author{
MAKOTO OHTSUKA
}

To Professor Kiyoshi Noshiro on the occasion of his 60th birthday

Introduction. The electric capacity of a conductor in the 3-dimensional euclidean space is defined as the ratio of a positive charge given to the conductor and the potential on its surface. The notion of capacity was defined mathematically first by N. Wiener [7] and developed by C. de la Vallée Poussin, O. Frostman and others. For the history we refer to Frostman's thesis [2]. Recently studies were made on different definitions of capacity and related notions. We refer to M. Ohtsuka [4] and G. Choquet [1], for instance. In the present paper we shall investigate further some relations among various kinds of capacity and related notions. A part of the results was announced in a lecture of the author in 1962.1)

1. Let $E$ and $F$ be locally compact Hausdorff spaces and $\Phi(x, y)$ be a lower semicontinuous function on $E \times F$, satisfying $-\infty<\Phi(x, y) \leqq \infty$. This function is called a kernel. As measures we shall consider only non-negative Radon measures with compact support in $E$ or in $F$. The potential $\int \Phi(x, y) d \mu(y)$ $\left(\int \Phi(x, y) d \nu(x)\right.$ resp.) of a measure $\mu$ ( $\nu$ resp.) will be denoted by $\Phi(x, \mu)(\Phi(\nu, y)$ resp.) and the double integral $\iint \Phi(x, y) d \mu(y) d \nu(x)=\int \Phi(x, \mu) d \nu(x)$ by $\Phi(\nu, \mu)$.

Let $X$ be any non-empty set in $E$ and $\mu$ be a measure in $F$. We set

$$
V(X, \mu)=\sup _{x \in X} \Phi(x, \mu) \quad \text { and } \quad U(X, \mu)=\inf _{x \in X} \Phi(x, \mu) .
$$

Let $Y$ be any non-empty set in $F$, and denote by $\mathscr{U}_{Y}$ the class of unit measures with compact support in $Y$. We put

Received August 11, 1966.

1) Capacity, Symposium on potential theory, Hakone, 1962. 


$$
V_{X}(Y)=\inf _{\mu \in \mathcal{U}_{Y}} V(X, \mu) \quad \text { and } \quad U_{X}(Y)=\sup _{\mu \in \mathcal{U}_{Y}} U(X, \mu) .
$$

Similarly we define $\check{V}_{Y}(X)$ and $\check{U}_{Y}(X)$ by $\inf _{\nu \in \mathcal{U}_{x}} \sup _{y \in Y} \Phi(\nu, y)$ and $\sup _{\nu \in \mathcal{U}_{X}} \inf _{y \in Y} \Phi(\nu, y)$ respectively. B. Fuglede [3] proved the identity $V_{E}(K)=\check{U}_{K}(E)$, where $K$ is a non-empty compact subset of $F$.

In the special case $E=F$ we set

$$
W_{i}(X)=\inf _{\mu \in \mathcal{U}_{X}} \Phi(\mu, \mu), V(X)=\inf _{\mu \in \mathcal{U}_{X}} V\left(S_{\mu}, \mu\right) \quad \text { and } \quad U(X)=\sup _{\mu \in \mathcal{U}_{X}} U\left(S_{\mu}, \mu\right) .
$$

If the adjoint kernel $\check{\Phi}(x, y)=\Phi(y, x)$ is considered, the corresponding quantities will be denoted by $\check{W}_{i}(X), \check{V}(X)$ and $\check{U}(X)$. We shall establish

Theorem 1. Suppose $E=F$ and let $K$ be a non-empty compact set in $E$. Then

$$
W_{i}(K)=\check{W}_{i}(K) \leqq V(K)=\check{V}(K) \leqq\left\{\begin{array}{l}
V_{K}(K)=\check{U}_{K}(K) \\
\check{V}_{K}(K)=U_{K}(K)
\end{array}\right\} \leqq\left\{\begin{array}{l}
V_{E}(K)=\check{U}_{K}(E) \\
\check{V}_{E}(K)=U_{K}(E) \\
U(K)=\check{U}(K)
\end{array}\right.
$$

and these relations can not be improved in general.

Proof. The equalities $V_{E}(K)=\check{U}_{K}(E)$ and $\check{V}_{E}(K)=U_{K}(E)$ are special cases of the above quoted identity due to Fuglede. The equalities $V_{K}(K)=\check{U}_{K}(K)$ and $\check{V}_{K}(K)=U_{K}(K)$ are further special cases. The equalities $V(K)=\check{V}(K)$ and $U(K)=\check{U}(K)$ were found by Ohtsuka [5]; cf. [6] too. It is evident that $W_{i}(K)=\check{W}_{i}(K)$. Thus all equalities are justified.

The inequality $W_{i}(K) \leqq V(K)$ follows from

$$
W_{i}(K) \leqq \int \Phi(x, \mu) d \mu(x) \leqq \sup _{x \in S_{\mu}} \Phi(x, \mu)
$$

which is valid for any $\mu \in \mathscr{U}_{K}$. The inequalities $V(K) \leqq V_{K}(K) \leqq V_{E}(K)$ and $U_{K}(K) \leqq U(K)$ are clear.

We shall give examples in which the inequalities are strict. Consider first the space $E$ consisting of two points $x_{1}$ and $x_{2}$. If the kernel $\Phi$ is given by the matrix $\left(\begin{array}{ll}1 & 1 \\ \frac{1}{2} & 1\end{array}\right)$, then $W_{i}(K)=7 / 8$ and $V(K)=1$ for $K=E$. If we consider the symmetric kernel given by $\left(\begin{array}{ll}1 & 2 \\ 2 & 1\end{array}\right), V(K)=1$ but $V_{K}(K)=3 / 2$. If $K$ consists of one point $x_{1}$ and $\Phi$ is given by $\left(\begin{array}{ll}1 & 2 \\ 2 & 1\end{array}\right)$, then $U(K)=V_{K}(K)=1$ but $V_{E}(K)=2$. 
If $K$ consists of two points and $\Phi$ is given by $\left(\begin{array}{ll}1 & 1 \\ 1 & 2\end{array}\right)$, then $V_{E}(K)=V_{K}(K)=1$ but $U(K)=2$. Our proof will be completed if we can find a kernel for which $V_{K}(K)<\check{V}_{K}(K)$. This is possible, because $V_{K}(K)=1$ but $\check{V}_{K}(K)=2$ for $K$ consisting of two points and $\Phi=\left(\begin{array}{ll}1 & 2 \\ 1 & 2\end{array}\right)$.

2. Suppose still $E=F$. We define $D_{n}(X)$ by

$$
\frac{1}{n(n-1)} \inf _{x_{1}, \ldots \ldots, x_{n} \in X} \sum_{i \neq j} \Phi\left(x_{i}, x_{j}\right) \text {. }
$$

This increases as $n \rightarrow \infty$. In fact, if we exclude the terms containing $x_{k}$ and denote the remaining sum by $\sum_{i \neq j}^{(k)}$, then

$$
\begin{gathered}
\sum_{i \neq j} \Phi\left(x_{i}, x_{j}\right)=\frac{1}{n-2} \sum_{k=1}^{n} \sum_{i \neq j}^{(k)} \Phi\left(x_{i}, x_{j}\right) \geqq \frac{1}{n-2} \sum_{k=1}^{n}(n-1)(n-2) D_{n-1}(X) \\
=n(n-1) D_{n-1}(X) .
\end{gathered}
$$

We set

$$
\lim _{n \rightarrow \infty} D_{n}(X)=D(X)
$$

It is a known result that $D(K)=W_{i}(K)$; see, for instance, Choquet [1]. In case $K$ is a compact set in $E_{3}$ and $\Phi$ is Newtonian, $1 / D(K)$ is called the transfinite diameter of $K$.

We come back to the general case where $E$ and $F$ may not be the same. Consider two non-empty sets $X$ and $Y$ in $E$ and $F$ respectively. We set

$$
n R_{n}(X, Y)=\sup _{y_{1}, \ldots \ldots, y_{n} \in Y} \inf _{x \in X} \sum_{i=1}^{n} \Phi\left(x, y_{i}\right)
$$

We shall assume $R_{1}(X, Y)>-\infty$ and show that $\lim _{n \rightarrow \infty} R_{n}(X, Y)$ exists. Choose $y_{1} \in Y$ such that $\inf _{x \in X} \Phi\left(x, y_{1}\right)>-\infty$. Then $n R_{n}(X, Y) \geqq \inf _{x \in X} n \Phi\left(x, y_{1}\right)>-\infty$. If $y_{1}, \ldots \ldots, y_{n}, \eta_{1}, \ldots \ldots, \eta_{m} \in Y$, then

$$
\begin{aligned}
(n+m) R_{n+m}(X, Y) & \geqq \inf _{x \in X}\left\{\sum_{i=1}^{n} \Phi\left(x, y_{i}\right)+\sum_{j=1}^{m} \Phi\left(x, \eta_{j}\right)\right\} \\
& \geqq \inf _{x \in X} \sum_{i=1}^{n} \Phi\left(x, y_{i}\right)+\inf _{x \in X} \sum_{j=1}^{m} \Phi\left(x, \eta_{j}\right),
\end{aligned}
$$

from which it follows that

$$
(n+m) R_{n+m}(X, Y) \geqq n R_{n}(X, Y)+m R_{m}(X, Y) .
$$


On the other hand,

$$
\begin{aligned}
n k R_{n}(X, Y) & =k \sup _{y_{1}, \cdots \ldots, y_{n} \in Y} \inf _{x \in X} \sum_{i=1}^{n} \Phi\left(x, y_{i}\right) \\
& \leqq \sup _{\eta_{1}, \cdots \cdots, \eta_{n k} \in Y} \inf _{x \in X} \sum_{j=1}^{n k} \Phi\left(x, \eta_{j}\right)=n k R_{n k}(X, Y) .
\end{aligned}
$$

Therefore

$$
(n k+m) R_{n k+m}(X, Y) \geqq n k R_{n k}(X, Y)+m R_{m}(X, Y) \geqq n k R_{n}(X, Y)+m R_{m}(X, Y)
$$

and hence

$$
R_{n k+m}(X, Y) \geqq \frac{n k}{n k+m} R_{n}(X, Y)+\frac{m}{n k+m} R_{m}(X, Y) .
$$

Given $\varepsilon, 0<\varepsilon<1$, we choose $n_{0}$ such that

$$
R_{n_{0}}(X, Y) \geqq \begin{cases}\varlimsup_{n \rightarrow \infty} R_{n}(X, Y)-\varepsilon & \text { if } \varlimsup_{n \rightarrow \infty} R_{n}(X, Y)<\infty, \\ 1 / \varepsilon & \text { if } \varlimsup_{n \rightarrow \infty} R_{n}(X, Y)=\infty .\end{cases}
$$

Next we choose $k_{0}$ such that, for any $k \geqq k_{0}$ and every $m(0 \leqq m \leqq n-1)$, it holds that

$$
\frac{n_{0} k}{n_{0} k+m}>1-\varepsilon \quad \text { and } \quad \frac{m}{n_{0} k+m} R_{m}(X, Y)>-\varepsilon .
$$

In case $\varlimsup_{n \rightarrow \infty} R_{n}(X, Y)=\infty,(1)$ yields

$$
R_{n_{0} k+m}(X, Y) \geqq \frac{1-\varepsilon}{\varepsilon}-\varepsilon
$$

for any $k \geqq k_{0}$ and every $m, 0 \leqq m \leqq n_{0}-1$. It follows that $\lim _{n \rightarrow \infty} R_{n}(X, Y)=\infty$.

In case $\varlimsup_{n \rightarrow \infty} R_{n}(X, Y)<\infty$, we choose $m_{k}\left(0 \leqq m_{k} \leqq n_{0}-1\right)$ such that

(2) $R_{n_{0} k+m_{k}}(X, Y) \leqq \begin{cases}\frac{\lim }{n \rightarrow \infty} R_{n}(X, Y)+\varepsilon & \text { if } \frac{\lim _{n \rightarrow \infty} R_{n}(X, Y)>-\infty}{-1 / \varepsilon}\end{cases}$

It holds on account of (1) that

$$
\lim _{k \rightarrow \infty} R_{n_{0} k+m_{k}}(X, Y) \geqq R_{n_{0}}(X, Y) \geqq \varlimsup_{n \rightarrow \infty} R_{n}(X, Y)-\varepsilon \cdot
$$


This and (2) yield $\lim _{n \rightarrow \infty} R_{n}(X, Y) \geqq \varlimsup_{n \rightarrow \infty} R_{n}(X, Y)$. Thus $\lim _{n \rightarrow \infty} R_{n}(X, Y)$ exists. We shall denote this limit by $R(X, Y)$.

Remark. There is an example in which $\lim _{n \rightarrow \infty} R_{n}(X, Y)$ does not exist. Take the $x$-axis as $X=E$ and $\{1,2, \ldots \ldots\}$ as $Y=F$. We define $\Phi(x, n)$ by $(-1)^{n} x$. Then $R_{n}(X, Y)=-\infty$ if $n$ is odd and $R_{n}(X, Y)=0$ if $n$ is even.

Let us establish

Theorem 2. Let $K$ be a non-empty compact set in $E$, and $Y$ be any non-empty set in $F$. Then $R(K, Y)$ exists and

$$
R(K, Y)=U_{K}(Y) .
$$

Proof. First we note that $R_{1}(K, Y)=\sup _{y \in Y} \inf _{x \in K} \Phi(x, y)>-\infty$, whence $R(K, Y)=\lim _{n \rightarrow \infty} R_{n}(K, Y)$ exists. For each $n$

$$
R_{n}(K, Y)=\frac{1}{n} \sup _{y_{1}, \ldots \ldots, y_{n} \in Y} \inf _{x \in K} \sum_{i=1}^{n} \Phi\left(x, y_{i}\right) \leqq U_{K}(Y),
$$

so that $R(K, Y) \leqq U_{K}(Y)$. To prove the inverse inequality take $\mu \in \mathscr{U}_{\mathrm{Y}}$. Given $\varepsilon>0$, we can find a continuous function $\Phi_{\varepsilon}(x, y)$ on $K \times S_{\mu}$ such that $\Phi_{\varepsilon}(x, y) \leqq \Phi(x, y)$ on $K \times S_{\mu}$ and

$$
\min _{x \in K} \Phi_{\varepsilon}(x, \mu) \geqq \min _{x \in K} \Phi(x, \mu)-\varepsilon .
$$

There exist a finite subdivision $S_{\mu}=\bigcup_{i=1}^{k} Y_{i}$ into mutually disjoint Borel sets $Y_{1}, \ldots \ldots$, $Y_{k}$ and points $y_{1} \in Y_{1}, \ldots \ldots, y_{k} \in Y_{k}$ such that

$$
\left|\Phi_{\varepsilon}(x, y)-\Phi_{\varepsilon}\left(x, y_{i}\right)\right|<\varepsilon
$$

whenever $x \in K$ and $y \in Y_{i}$ for each $i$. We have

$$
\left|\sum_{i} \Phi_{\varepsilon}\left(x, y_{i}\right) \mu\left(Y_{i}\right)-\Phi_{\varepsilon}(x, \mu)\right| \leqq \sum_{i} \int_{Y_{i}}\left|\Phi_{\varepsilon}\left(x, y_{i}\right)-\Phi_{\varepsilon}(x, y)\right| d \mu(y) \leqq \varepsilon
$$

on $K$ and hence

$$
\min _{x \in K} \sum_{i} \Phi_{\varepsilon}\left(x, y_{i}\right) \mu\left(Y_{i}\right) \geqq \min _{x \in K} \Phi_{\varepsilon}(x, \mu)-\varepsilon \geqq \min _{x \in K} \Phi(x, \mu)-2 \varepsilon .
$$

We approximate each $\mu\left(Y_{i}\right)$ by a non-negative rational number $r_{i}$ such that $\sum_{i} r_{i}=1$ and 


$$
\min _{x \in K} \sum_{i} \Phi_{\varepsilon}\left(x, y_{i}\right) \mu\left(Y_{i}\right) \leqq \min _{x \in K} \sum_{i} \Phi_{\varepsilon}\left(x, y_{i}\right) r_{i}+\varepsilon \leqq \min _{x \in K} \sum_{i} \Phi\left(x, y_{i}\right) r_{i}+\varepsilon
$$

Set $r_{i}=p_{i} / q$ with integers $p_{i} \geqq 0$ and $q>0$, and consider

$$
\frac{1}{q}\left\{p_{1} \Phi\left(x, y_{1}\right)+p_{2} \Phi\left(x, y_{2}\right)+\ldots \ldots+p_{k} \Phi\left(x, y_{k}\right)\right\}
$$

Its minimum on $K$ is not greater than $R_{q}(K, Y)$. Thus

$$
\min _{x \in K} \Phi(x, \mu) \leqq R_{q}(K, Y)+3 \varepsilon \text {. }
$$

Since we can take $q$ arbitrarily large, $\min _{x \in K} \Phi(x, \mu) \leqq R(K, Y)+3 \varepsilon$, whence $\min _{x \in K} \Phi(x, \mu) \leqq R(K, Y)$. Because of the arbitrariness of $\mu \in \mathscr{U}_{\mathrm{Y}}$, we have $U_{K}(Y) \leqq R(K, Y)$, which gives the equality.

3. Finally we prove

Theorem 3. Let $X$ be a non-empty set in $E$ and $L$ be a non-empty compact set in $F$. In order that there be $\mu \in \mathscr{U}_{L}$ such that $\Phi(x, \mu)=\infty$ for every $x \in X$, it is necessary and sufficient that $U_{X}(L)=\infty$.

Proof. Suppose that there is a measure $\mu \in \mathscr{U}_{L}$ such that $\Phi(x, \mu)=\infty$ for every $x \in X$. Then

$$
U_{X}(L)=\sup _{\mu \in \mathfrak{U}_{L}} \inf _{x \in X} \Phi(x, \mu)=\infty .
$$

Conversely assume $U_{X}(L)=\infty$. For each $k$ there is $\mu_{k} \in \mathscr{U}_{L}$ such that $\Phi\left(x, \mu_{k}\right)>2^{k}$ on $X . \quad$ Naturally $\sum_{k=1}^{\infty} 2^{-k} \mu_{k} \in \mathscr{U}_{L}$ and

$$
\Phi\left(x, \sum_{k=1}^{\infty} 2^{-k} \mu_{k}\right)=\infty \quad \text { for every } x \in X .
$$

Using Theorem 2 we obtain the following generalization of the so-called Evans-Selberg's theorem.

Corollary. Let $K$ and $L$ be non-empty compact sets in $E$ and $F$ respectively. In order that there be $\mu \in \mathscr{U}_{L}$ such that $\Phi(x, \mu)=\infty$ for every $x \in K$, it is necessary and sufficient that $R(K, L)=\infty$. 


\section{REFERENCES}

[1] G. Choquet: Diamètre transfini et comparaison de diverses capacités, Sém. Théorie du potentiel, $3(1958 / 59), \mathrm{n}^{\circ} 4,7 \mathrm{pp}$.

[2] O. Frostman: Potentiel d'équilibre et capacité des ensembles, Thèse, Lund, 1935, 118 pp.

[3] B. Fuglede: Le théorème du minimax et la théorie fine du potentiel, Ann. Inst. Fourier, 15 (1965), pp. 65-87.

[4] M. Ohtsuka: Selected topics in function theory, Tokyo, 1957, in Japanese.

[5] M. Ohtsuka: An application of the minimax theorem to the theory of capacity, J. Sci. Hiroshima Univ. Ser. A-I Math., 29 (1965), pp. 217-221.

[6] M. Ohtsuka: Generalized capacity and duality theorem in linear programming, ibid., 30 (1966), pp. 45-56.

[7] N. Wiener: Certain notions in potential theory, J. Math. Phys. M.I.T., 3 (1924), pp. 24-51.

Department of Mathematics, Faculty of Science, Hiroshima University 